\title{
Triply resonant coherent four-wave mixing in silicon nitride microresonators
}

\author{
Attila Fülöp ${ }^{1,}$, Clemens J. KrüCKel ${ }^{1}$, David Castelló-Lurbe ${ }^{2,3}$, Enrique Silvestre ${ }^{2}$, AND \\ VICTOR TORRES-COMPANY ${ }^{1}$ \\ ${ }^{1}$ Photonics Laboratory, Department of Microtechnology and Nanoscience (MC2), Chalmers University of Technology, Göteborg, Sweden \\ ${ }^{2}$ Departament d'Òptica, Universitat de València, Burjassot, Spain \\ ${ }^{3}$ Brussels Photonics Team (B-PHOT), Department of Applied Physics and Photonics (IR-TONA), Vrije Universiteit Brussel, B-1050 Brussel, Belgium \\ ${ }^{*}$ Corresponding author: attila.fulop@chalmers.se
}

Compiled August 26, 2015

The generation of multiple tones using four-wave mixing (FWM) has been exploited for many applications, ranging from wavelength conversion to frequency comb generation. FWM is a coherent process, meaning that its dynamics strongly depends on the relative phase among the waves involved. The coherent nature of FWM has been exploited for phasesensitive processing in different waveguide structures, but it has never been studied in integrated microresonators. Waveguides arranged in a resonant way allow for an effective increase in the wavelength conversion efficiency (at the expense of a reduction in the operational bandwidth). In this letter, we show that phase shaping of a three-wave pump provides an extra degree of freedom for controlling the FWM dynamics in microresonators. We present experimental results in single-mode, normal-dispersion high-Q silicon nitride resonators, and numerical calculations of systems operating in the anomalous dispersion regime. Our results indicate that the wavelength conversion efficiency and modulation instability gain in microcavities pumped by multiple waves can be significantly modified with the aid of simple lossless coherent control techniques.

OCIS codes: (130.3120) Integrated optics devices; (190.4380) Nonlinear optics, four-wave mixing; (230.5750) Resonators.

\section{http://dx.doi.org/10.1364/OL.XX.XXXXXX}

The effect of four-wave mixing (FWM) has enabled a variety of ultrafast photonics applications, including high-speed sampling, switching, wavelength conversion and amplification [1,2]. The FWM effect can become very efficient when using resonating systems [3], when the input waves are matched to the resonator cavity's longitudinal modes. This comes at the expense of a reduction in the operational bandwidth owing to an inherent tradeoff between resonance linewidth and conversion efficiency (although this can be partly alleviated by using an arrangement of coupled resonators [4]). Nevertheless, resonant FWM has been used in practice for wavelength conversion of high-speed data signals [4] and threshold-less comb generation with two $[5,6]$ and several pumps [7]. Synchronous pumping of nonlinear fiber loops has been used for optical data buffering [8]. Nonlinear effects including the breaking of time-reversal symmetry [9] and the generation of dispersive waves [10] have been reported in fiber cavities.

If three or more waves are input to the nonlinear medium, the gain dynamics of the mixing process, be it resonant or not, depends on their relative phases [11]. While this phase-sensitive process has been widely studied in different fiber configurations $[11,12]$, the phase sensitive dynamics of FWM in resonators has received less attention outside of single pump cases [13].

This letter explores the FWM phase dependence both in experiments and simulations for integrated resonating cavity systems. The first part focuses on the phase sensitive nature of non-degenerate FWM and compares experiments with simulations as well as a simplified analytical model. The second part shows, through simulations, the strongly phase-dependent nature of the idlers generated in the modulation instability (MI) regime, where the nonlinear medium has anomalous dispersion. Our results indicate that control over the relative phase of the initial seed provides a powerful tool that can greatly influence the generated waveform at the system output. Optimization of that phase will therefore be critical for frequency comb generation in both normal and anomalous dispersion regimes.

In the experiment, a silicon nitride based microresonator with a $Q$-value above $7 \times 10^{5}$ was pumped close to resonance by three phase-locked waves of identical amplitude at $1547.9940 \mathrm{~nm}$, $1548.2260 \mathrm{~nm}$ and $1548.4582 \mathrm{~nm}$ with a total pump power of $25 \mathrm{dBm}$ giving an estimated coupled power (to the bus waveguide) of $21 \mathrm{dBm}$. The pumps were generated using a single tunable external cavity laser with a specified linewidth of $100 \mathrm{kHz}$ and an intensity modulator driven by a radio frequency source set to match the free spectral range (FSR) of the resonator cavity at roughly $29 \mathrm{GHz}$. A pulse shaper (with $10 \mathrm{GHz}$ optical resolution) was then used to tune the phase of the central pump wave. Due to FWM, new lines were then generated inside the microresonator [See Fig. 1(a)]. A schematic of the setup can be seen in Fig. 1(b). The silicon nitride microresonators used in this experiment were manufactured in a multi-project wafer run using the commercially available dual-layer Triplex ${ }^{\mathrm{TM}}$ technology [14]. The 

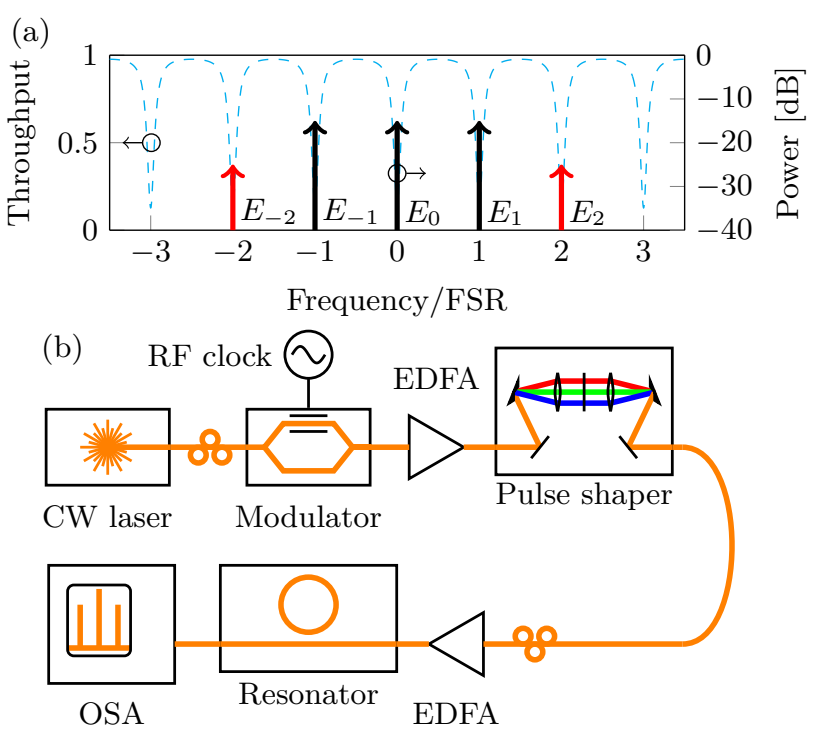

Fig. 1. (a) A sketch showing the three pumps (black) and the generated idler lines (red) in the foreground with a transmission spectrum showing the relevant resonances in the background. (b) A schematic representation of the setup showing the initial generation block, containing the laser, the modulator and the pulse shaper, as well as the resonator. The two polarization controllers are tuned for optimum operation: the first one for maximum extinction in the intensity modulator and the second one for coupling into the TE mode of the device.

dual-layer technology allows for single mode operation while maintaining measured linear losses below $0.5 \mathrm{~dB} / \mathrm{cm}$. Since silicon nitride, as opposed to pure silicon, lacks two-photon absorption in the optical C-band, it is very promising for highpower applications [15]. Simulations done using a finite element solver (COMSOL) both verify the single mode property as well as provide an estimated value for the group velocity dispersion. Figure 2 shows the geometry as well as the corresponding simulated mode profile and dispersion curve. It is clearly visible that the waveguide has strong normal dispersion.

To verify the single mode property in practice, a transmission spectrum was measured by sweeping a single tunable external cavity laser over a wavelength range covering several FSRs. A part of that scan can be seen in Fig. 3(a). The broadband scan indicates that there exists no coupling to counterpropagating modes nor higher order transverse modes. This ensures that the normal dispersion profile is maintained across the C-and L-band. This is in contrast to recent experiments where interactions between modes produce abrupt changes in the local dispersion of the microresonator [16, 17]. Figure 3(b) shows a zoomed in view of one resonance. When the pump power is increased, owing to thermal effects, the resonance will gradually shift towards the red side when scanning the pump wavelength giving an asymmetric transmission spectrum. This leads to a practical difficulty of pumping close to resonance on the red side, with the detuning parameter $\delta_{0}>0$, but also contributes to thermal stability when pumping on the blue side, where $\delta_{0}<0$ [18]. We will therefore, even in the simulations, stick to situations where $\delta_{0}<0$. In practice, this also means that it is challenging to achieve phase matching and induce modulation instability in this waveguide platform when pumping with a single CW laser.

Sweeping the phase of the middle pump with the pulse shaper resulted in the generated idler power varying periodically, matching numerical simulations, see Fig. 4 . The simulations were done using a standard split-step Fourier nonlinear Schrödinger equation (NLSE) solver to propagate the light inside the resonator while coupling in the pumps together with quantum noise after every round-trip. In the simulations, the value for the detuning, $\delta_{0}$, was approximated to be -0.025 by matching a measured value for the outcoupled idler power compared to the outcoupled pump power with $\phi_{\mathrm{s}}=0$ to the corresponding simulated value. The resulting value for $\delta_{0}$ was then kept for the rest of the simulations and numerical calculations. The results from the measurements show a $\pi$-periodic power dependence on the central pump phase as well as a variation in the conversion efficiency of $8.0 \mathrm{~dB}$. The split-step simulations confirm the periodicity while having a slightly larger variation at $8.4 \mathrm{~dB}$.

To get a more quantitative understanding of the influence of the resonator parameters in the final idler power, we study the stationary solutions of the Lugiato-Lefever equation [19]. This equation has previously been used in describing the nonlinear dynamics of both fiber-based and microresonator systems [20, 21]:

$$
\begin{aligned}
0= & \left(-\frac{\alpha+\theta}{2}-i \delta_{0}\right) E_{\mathrm{S}}(\tau)-i L \frac{\beta_{2}}{2} \frac{\partial^{2} E_{\mathrm{S}}(\tau)}{\partial \tau^{2}}+ \\
& i \gamma L E_{\mathrm{S}}(\tau)\left|E_{\mathrm{S}}(\tau)\right|^{2}+\sqrt{\theta} E_{\mathrm{in}}(\tau),
\end{aligned}
$$

where the constants $L, \alpha=\alpha_{\mathrm{i}} L, \theta, \beta_{2}$ and $\gamma$ stand for the resonator length, the total power loss during one round trip, the power coupling coefficient, the group velocity dispersion and the nonlinearity respectively. In the experiment, we observed the generation of two new lines on either side of the pumps, so we are interested in stationary solutions, $E_{\mathrm{S}}(\tau)$, consisting of five waves:

$$
E_{\mathrm{S}}(\tau)=\sum_{m=-2}^{2} E_{m} \exp (m i \Omega \tau),
$$

where $E_{m}$ represents the complex amplitude of wave $m$. The frequency spacing between the waves is denoted with $\Omega$ and corresponds to the FSR of the resonator. For the input signal $E_{\text {in }}(\tau)$, we specify the three-wave pump:

$$
E_{\mathrm{in}}(\tau)=E_{\mathrm{p}}\left(\exp (-i \Omega \tau)+\exp \left(i \phi_{\mathrm{s}}\right)+\exp (i \Omega \tau)\right),
$$

where the amplitudes of the three waves are all equal, $\left|E_{\mathrm{p}}\right|$, the phases of the two side-pumps are fixed and equal, and the phase of the middle pump wave is shifted by $\phi_{\mathrm{s}}$ (in practice controlled by the pulse shaper). We can do this without loss of

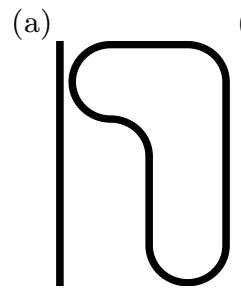

(b)
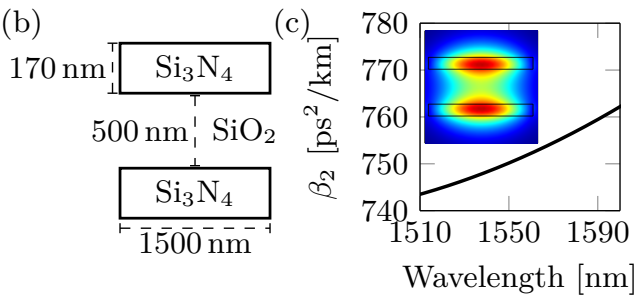

Fig. 2. (a) A top view of the layout of the resonator, with a bus waveguide-resonator gap of $1000 \mathrm{~nm}$ and a total length of $6031.86 \mu \mathrm{m}$. (b) A schematic of the 2D cross section of the waveguide geometry. (c) Dispersion simulation around $1550 \mathrm{~nm}$ for the fundamental TE mode with the inset showing the simulated power distribution of the mode. 


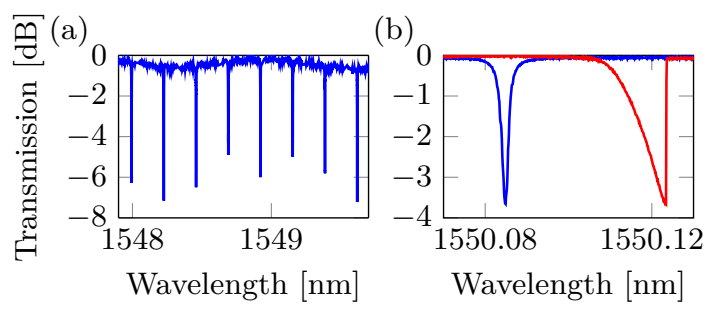

Fig. 3. (a) Transmission spectrum showing multiple resonances belonging to the single propagating mode. (b) Zoomed in view showing one resonance swept with a $5 \mathrm{dBm}$ low powered pump (blue) and a $25 \mathrm{dBm}$ high powered pump (red). Owing to temperature effects occurring when pumping with high powers the resonance experiences a shift towards longer wavelengths leading to a triangular shape when sweeping[18].

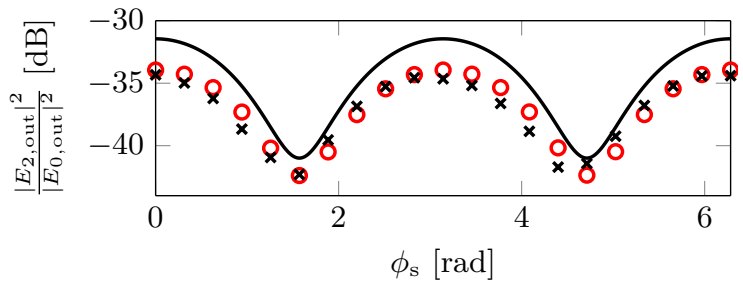

Fig. 4. Outcoupled idler power compared to the outcoupled pump power as a function of the central pump phase, $\phi_{\mathrm{s}}$. The solid black curve shows the calculated ratio using the simplified analytical formula, while the red rings and the black crosses show the simulated and measured ratios respectively. The offset between the analytical formula and the measured and simulated data is present because of the extra assumptions that are made during its derivation.

generality, since a linear phase relation between the three lines only corresponds to a time shift of the resulting waveform. By inserting Eqs. (2) and (3) back into Eq. (1) and discarding the terms containing frequencies more than two FSRs away from the central pump we arrive at a set of 5 complex valued equations, one for each line. The ones describing the idlers are as follows:

$$
\begin{aligned}
0 & =E_{ \pm 2}\left(-i \frac{\alpha+\theta}{2}+\delta_{0}-2 L \gamma P_{\text {tot }}+L \gamma\left|E_{ \pm 2}\right|^{2}-2 L \beta_{2} \Omega^{2}\right) \\
& -L \gamma\left(E_{0}^{*} E_{ \pm 1}^{2}+E_{0}^{2} E_{\mp 2}^{*}+2 E_{0} E_{ \pm 1} E_{\mp 1}^{*}+2 E_{-1} E_{1} E_{\mp 2}^{*}\right)
\end{aligned}
$$

where $P_{\text {tot }}=\left|E_{0}\right|^{2}+\left|E_{-1}\right|^{2}+\left|E_{1}\right|^{2}+\left|E_{-2}\right|^{2}+\left|E_{2}\right|^{2}$.

To draw some qualitative conclusions about how the idler power depends on various parameters, we will have to make some extra assumptions and simplifications. When pumping a nonlinear material with three pumps there are several FWM processes acting simultaneously. These will correspond to different terms in the equation above. Some of the processes have already been ignored when avoiding the frequency terms far away from the pumps, where the power levels are low [22]. We will from here on also ignore the two symmetric FWM processes that couple the two idlers together: the degenerate one transferring power from the middle pump to the two idlers and the nondegenerate one transferring power from the two side pumps to the two idlers. These assumptions, removing the terms containing $E_{\mp 2}^{*}$, allow us to simplify Eq. (4) retaining the main features of the system behavior, and permit recasting it into the following closed-form expression:

$$
\begin{aligned}
\left|E_{ \pm 2}\right|^{2} & =\frac{\left|E_{0}\right|^{2}\left|E_{-1}\right|^{2}\left|E_{1}\right|^{2} L^{2} \gamma^{2}}{\frac{(\alpha+\theta)^{2}}{4}+\left(\delta_{0}-2 L \gamma P_{\text {tot }}+L \gamma\left|E_{ \pm 2}\right|^{2}-2 L \beta_{2} \Omega^{2}\right)^{2}} \\
& \times\left(4+\frac{\left|E_{ \pm 1}\right|^{2}}{\left|E_{\mp 1}\right|^{2}}+4 \frac{\left|E_{ \pm 1}\right|}{\left|E_{\mp 1}\right|} \cos \left(2 \phi_{0}-\phi_{-1}-\phi_{1}\right)\right) .
\end{aligned}
$$

Further assuming that the idlers will remain low in power, $E_{0, \pm 1} \gg E_{ \pm 2}$, and that the three pumped waves remain undepleted and equal in power (as it seems to be in the experiment), we get:

$$
\begin{aligned}
\left|E_{0}\right|^{2} & =\left|E_{ \pm 1}\right|^{2} \approx \frac{\left|E_{\mathrm{p}}\right|^{2} \theta}{\frac{(\alpha+\theta)^{2}}{4}+\delta_{0}^{2}}, \\
\phi_{0} & =\phi_{-1}+\phi_{\mathrm{s}}=\phi_{1}+\phi_{\mathrm{s}},
\end{aligned}
$$

leading to a closed form expression for $\left|E_{ \pm 2}\right|^{2}$ that only depends on the initially known parameters of the system:

$$
\left|E_{ \pm 2}\right|^{2}=\frac{\left|E_{0}\right|^{6} L^{2} \gamma^{2}\left(5+4 \cos \left(2 \phi_{\mathrm{s}}\right)\right)}{\frac{(\alpha+\theta)^{2}}{4}+\left(\delta_{0}-6\left|E_{0}\right|^{2} L \gamma-2 L \beta_{2} \Omega^{2}\right)^{2}}
$$

where $\left|E_{0}\right|^{2}$ is given in Eq. (6). The pump phase dependent conversion efficiency for $\left|E_{ \pm 2}\left(\phi_{\mathrm{s}}\right)\right|^{2}$ is thus 9 (or $9.5 \mathrm{~dB}$ ) with a period of $\pi$ matching the measured and simulated curves seen in Fig. 4. What is interesting to note from this analysis, is that the maximum conversion efficiency will take place for a phase matching condition similar to the case of modulation instability in microresonator frequency combs pumped by a single $\mathrm{CW}$ laser [23].

Although strictly speaking there is no threshold in the case of FWM wavelength conversion in a resonator pumped with several waves [5], one can see that the efficiency of the process is inversely proportional to the square of the resonator loss. For comparison, a similar analysis can be carried out for a simple waveguide system without a resonating cavity. In that case, the steady state situation would be equivalent to a propagated distance that is long enough for the gains from the Kerr effect and the losses to be of equal strength in the idler, but short enough for the losses not to have attenuated the pumps significantly yet. By performing the same simplifications as before, we arrive at the following equation:

$$
\left|E_{ \pm 2, \mathrm{wg}}\right|^{2}=\frac{\left|E_{\mathrm{p}}\right|^{6} \gamma^{2}\left(5+4 \cos \left(2 \phi_{\mathrm{s}}\right)\right)}{\frac{\alpha_{i}^{2}}{4}+\left(6\left|E_{\mathrm{p}}\right|^{2} \gamma+2 \beta_{2} \Omega^{2}\right)^{2}}
$$

where $\alpha_{\mathrm{i}}$ corresponds to the propagation losses per length while the rest of the parameters are defined as before. The significant difference between the ring and the waveguide case is that for the waveguide it is the pump power, whereas for the resonator system it is the in-cavity power that appears. To draw some conclusions, we can take an additional step: assume that, for the resonating system, $\delta_{0}=0$, the dispersion is optimal and that we have critical coupling $(\theta=\alpha)$. The in-cavity idler power will then be proportional to $1 / \alpha^{5}$ and the outcoupled one will be proportional to $1 / \alpha^{4}$. This is in comparison with the straight waveguide case, where the output idler power will remain proportional to $1 / \alpha_{\mathrm{i}}^{2}$. In the low loss regime, pumping a resonator instead of a waveguide will thus greatly enhance the output idler power.

The previous analysis dealt with resonant wavelength conversion via FWM. However, the thermal locking dynamics together with the normal dispersion of the waveguide prevented 


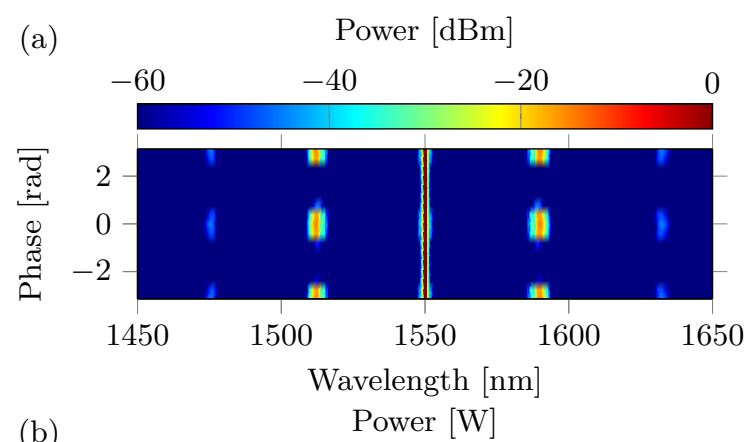

(b)

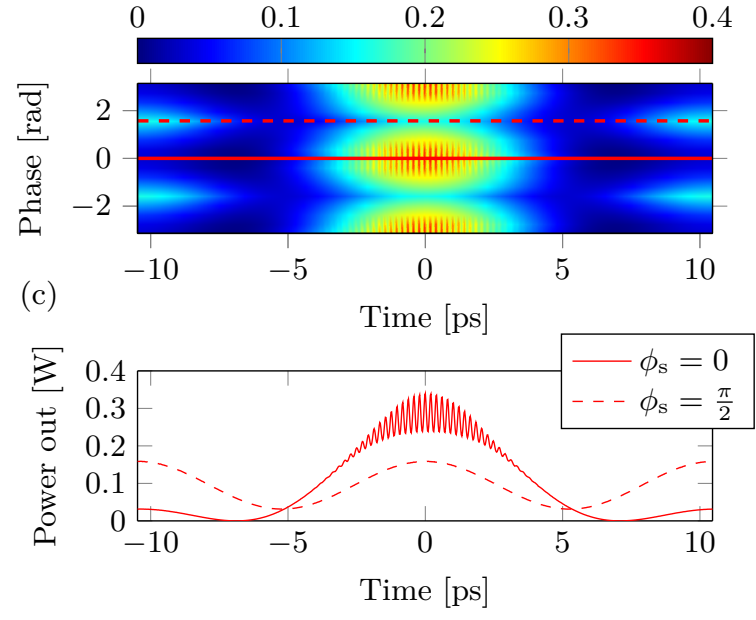

Fig. 5. (a) Simulated output spectra for a microresonator pumped with three equally powered waves at resonance as a function of the middle pump phase. The parameters were set to $P_{\text {in,tot }}=0.1 \mathrm{~W}, \alpha=\theta=0.01, r=500 \mu \mathrm{m}, \delta_{0}=-0.05$, $n_{\mathrm{g}}($ at $1550 \mathrm{~nm})=2, \gamma=5 / \mathrm{W} / \mathrm{m}$ and $\beta_{2}=-50 \mathrm{ps}^{2} / \mathrm{km}$. The simulation resolution corresponds to 1 FSR so the spacing between the pump lines is not resolved in the figure. $(b-c)$ Simulated output power in the time domain for the same parameters with a detailed view of two time domain traces.

the formation of MI and hence parametric gain. In the anomalous dispersion regime, when the power levels are high enough, combs can be generated using a single continuous wave pump (see analysis in [20, 21, 24]). To investigate the phase dependence in such a case, we turn to the split-step NLSE simulations. The analytical model developed in the previous paragraph will not be valid in general as we discarded the waves located far away from the pump in the frequency domain. When using several pumps to seed the comb, lower power levels can already cause MI growth $[5,7]$. In these cases however, the relative pump phases also have great effect on the final power in the MI sidebands. Figure 5 shows the results from a simulation demonstrating such a case. The simulations were done using three equally powered pumps, placed slightly off resonance on the blue, thermally stable, side. The ring parameters were chosen to be realistic and are specified in the figure caption. Setting the pump phase difference from $\phi_{\mathrm{s}}=0$ to $\phi_{\mathrm{s}}=\pi / 2$ gives significantly different results; in the first case, we see MI sidebands of significant amplitudes, whereas in the second case there is no new frequency generation at all, giving a phase dependent relative conversion efficiency for the generated idlers of above $40 \mathrm{~dB}$. In the time domain picture, when the MI gain is present, an interference pattern corresponding to the distance between the pump laser and the MI generated sidebands is also visible. These results indicate that the relative phase between the input lines drastically alter the gain profile of the MI. Further investigations are needed to find the limits and the full extent of this dependence.

In summary, we have demonstrated triply resonant fourwave mixing in a silicon nitride microresonator and verified the periodic phase dependence of the results using both split-step NLSE simulations and a simplified analytical expression derived from the Lugiato-Lefever model. We have also demonstrated simulations showing a strong phase dependence of modulation instability gain giving rise to a conversion efficiency difference of above $40 \mathrm{~dB}$ in resonators with anomalous dispersion. The control and optimization of the relative phases of a multiplepump seed thus provides a powerful tool in controlling the final waveform, with implications for applications both in the normal and the anomalous dispersion regime.

This project was supported by the Swedish Research Council (VR), the Spanish government (research project TEC201346643-C2-1-R), and the European Research Council under grant agreement ERC-2011-AdG - 291618 PSOPA.

\section{REFERENCES}

1. G. P. Agrawal, in "Applications of Nonlinear Fiber Optics," (Academic Press, 2008), chap. 8, p. 349, 2nd ed.

2. J. Hansryd, P. A. Andrekson, M. Westlund, L. Jie, and P. O. Hedekvist, IEEE J. Sel. Top. Quantum Electron. 8, 506 (2002).

3. A. C. Turner, M. A. Foster, A. L. Gaeta, and M. Lipson, Opt. Express 16, 4881 (2008).

4. F. Morichetti, A. Canciamilla, C. Ferrari, A. Samarelli, M. Sorel, and A. Melloni, Nat. Commun. 2, 296 (2011).

5. D. Strekalov and N. Yu, Phys. Rev. A 79 (2009).

6. T. Hansson and S. Wabnitz, Phys. Rev. A 90 (2014).

7. S. B. Papp, P. Del'Haye, and S. A. Diddams, Opt. Express 21, 17615 (2013).

8. F. Leo, S. Coen, P. Kockaert, S.-P. Gorza, P. Emplit, and M. Haelterman, Nature Photon. 4, 471 (2010).

9. Y. Xu and S. Coen, Opt. Lett. 39, 3492 (2014).

10. K. Luo, Y. Xu, M. Erkintalo, and S. G. Murdoch, Opt. Lett. 40, 427 (2015).

11. C. Lundström, Z. Tong, M. Karlsson, and P. A. Andrekson, Opt. Lett. 36, 4356 (2011).

12. C. J. McKinstrie and S. Radic, Opt. Express 12, 4973 (2004).

13. L. Maleki, V. S. Ilchenko, A. A. Savchenkov, W. Liang, D. Seidel, and A. B. Matsko, "High performance, miniature hyper-parametric microwave photonic oscillator," in "FCS, 2010 IEEE International," (IEEE), p. 558.

14. F. Morichetti, A. Melloni, M. Martinelli, R. G. Heideman, A. Leinse, D. H. Geuzebroek, and A. Borreman, J. Lightwave Technol. 25, 2579 (2007).

15. J. S. Levy, A. Gondarenko, M. A. Foster, A. C. Turner-Foster, A. L. Gaeta, and M. Lipson, Nature Photon. 4, 37 (2009).

16. T. Herr, V. Brasch, J. D. Jost, I. Mirgorodskiy, G. Lihachev, M. L. Gorodetsky, and T. J. Kippenberg, Phys. Rev. Lett. 113 (2014).

17. Y. Liu, Y. Xuan, X. Xue, P.-H. Wang, S. Chen, A. J. Metcalf, J. Wang, D. E. Leaird, M. Qi, and A. M. Weiner, Optica 1, 137 (2014).

18. T. Carmon, L. Yang, and K. J. Vahala, Opt. Express 12, 4742 (2004).

19. L. Lugiato and R. Lefever, Phys. Rev. Lett. 58, 2209 (1987).

20. M. Haelterman, S. Trillo, and S. Wabnitz, Opt Commun 91, 401 (1992).

21. S. Coen, H. G. Randle, T. Sylvestre, and M. Erkintalo, Opt. Lett. 38, 37 (2013).

22. G. P. Agrawal, in "Nonlinear Fiber Optics," (Academic Press, 2013), chap. 10 , p. 397,5 th ed.

23. V. Torres-Company, D. Castelló-Lurbe, and E. Silvestre, Opt. Express 22, 4678 (2014).

24. T. J. Kippenberg, R. Holzwarth, and S. A. Diddams, Science 332, 555 (2011). 